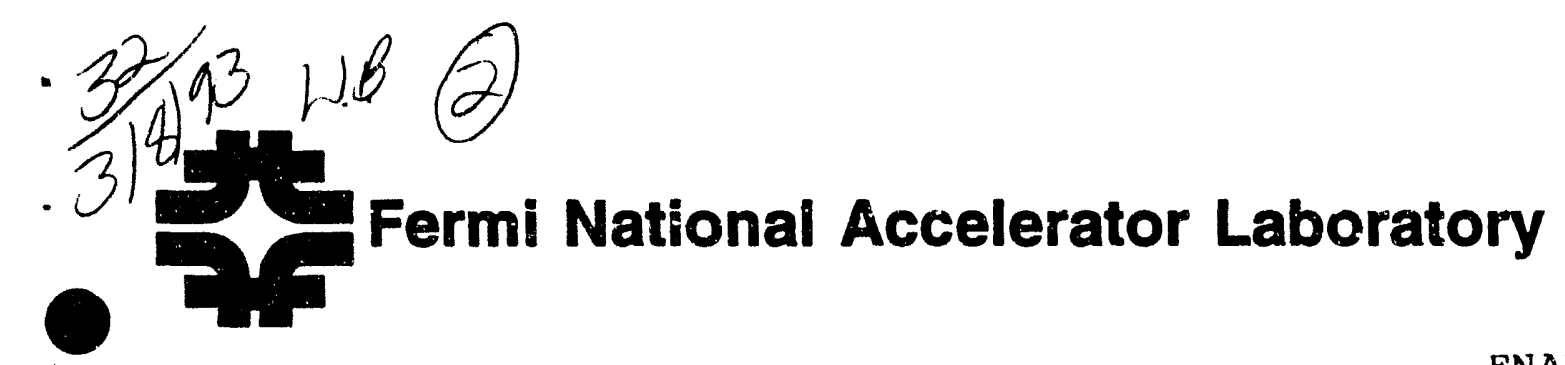

FNAL-TM-- 1821

DE93 008364

\title{
A Feedback Microprocessor for Hadron Colliders
}

\author{
D.A. Herrup, L. Chapman, A. Franck, T. Groves and B. Lublinsky \\ Fermi National Accelerator Laboratory \\ P.O. Box 500, Batavia, Illinois 60510
}

\section{DISCLAIMER}

\begin{abstract}
This report was prepared as an account of work sponsored by an agency of the United States Government. Neither the United States Government nor any agency thereof, nor any of their employees, makes any warranty, express or implied, or assumes any legal liability , product, or bility for the accuracy, completeness, or usefulness of any information, appand rights. Referprocess disclosed, or represents that its use would not infringe privaty by trade name, trademark, ence herein to any specific commercial product, process, or service by trade ndorsement, recommanufacturer, or otherwise does not necessarily constitute or imply agency thereof. The views mendation, or favoring by the United States Government or any agency thereof. The the and opinions of authors expressed herein do not United States Government or any agency thereof.
\end{abstract}

December 1992

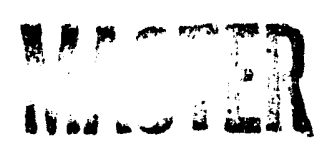

Submitted to isuc $3 r$ Instruments and Methods 


\section{Disclaimer}

This report was prepared as an account of work sponsored by an agency of the United States Government. Neither the United States Government nor any agency thereof, nor any of their employees, makes any warranty, express or implied, or assumes any legal liability or responsibility for the accuracy, completeness, or usefulness of any information, apparatus, product, or process disclosed, or represents that its use would not infringe privately owned rights. Reference herein to any specific commercial product, process, or service by trade name, trademark, manufacturer, or otherwise, does not necessarily constitute or imply its endorsement, recommendation, or favoring by the United States Government or any agency thereof. The views and opinions of authors expressed herein do not necessarily state or reflect those of the United States Government or any agency thereof. 


\title{
A Feedback Microprocessor for Hadron Colliders
}

\author{
D.A. Herrup, L. Chapman, A. Franck, T. Groves, B. Lublinsky \\ Fermi National Accelerator Laboratory, Batavia, IL
}

\begin{abstract}
A feedback microprocessor has been built for the TEVATRON. It has been constructed to be applicable to hadron colliders in general. Its inputs are realtime accelerator measurements, data describing the state of the TEVATRON, and ramp tables. The microprocessor software includes a finite state machine. Each state corresponds to a specific TEVATRON operation and has a state-specific TEVATRON model. Transitions between states are initiated by the global TEVATRON clock. Each state includes a cyclic routine which is called periodically and where all calculations are performed. The output corrections are inserted onto a fast TEVATRON-wide link from which the power supplies will read the realtime corrections. We also store all of the input data and output corrections in a set of buffers which can easily be retrieved for diagnostic analysis. In this paper we will describe this device and its use to control the TEVATRON tunes as well as other possible applications.
\end{abstract}

Introduction

In hadron colliders such as the Fermilab TEVATRON, the HERA $p$ ring, the SSC and the LHC, a well-defined sequence of operations is needed to take the accelerator from an initial state, without stored beam, to a final state, with stored beam and collisions at the interaction points. There are many intermediate states (such as acceleration), during which accelerator parameters change. It is difficult to construct a single model of the accelerator which applies during all these states. Instead, it is convenient to consider each process separately and construct models for each state. The entire process may be thought of as a finite state machine, with each state corresponding to a difierent operation and having its own model of the accelerator. In addition, the time scales at which the accelerator parameters change vary from state to state. Changes in accelerator parameters lead to variations in the beam parameters such as the tunes, coupling, and chromaticities. If the beam parameters are not carefully controlled, beam quality will deteriorate. One of the principal challenges of collider 
operations is to maintain high beam intensities and low emittances through the chain of operations from injection to collisions.

The sequence of operations in the TEVATRON is illustrative of this process ${ }^{1}$ (see Fig. 1). Operations proceed through the following (simplified) set of states: $p$ injection, energizing electrostatic separators to create different helical orbits for $p$ 's and $\bar{p}$ 's, $\bar{p}$ injection, acceleration, lattice modifications to produce low $\beta^{* 6} s$ at the interaction regions, and energizing additional separators to create head-on collisions at the two interaction regions. In $p$ and $\bar{p}$ injection, a closed orbit time bump moves the circulating orbit near an injection kicker, and back immediately after injection. Closed orbit changes may be accompanied by betatron tune and coupling changes caused by the non-linear fields of the bending dipoles and sextupoles. Energizing the separators has the same effect. During acceleration, all the magnetic elements are ramped. Although the linear lattice is kept constant, the multipoles change, affecting the tunes and coupling. The closed orbit may also vary. "Squeezing" is the term used to describe lattice changes to create small $\beta^{*}$ 's at the interaction regions. In principle, only a restricted set of quadrupoles should be needed for this step. However, since the quadrupole strengths are not precisely known, the exact settings for the quadrupoles cannot always be calculated. The closed orbit may not pass through the centers of the quadrupoles, leading to closed orbit (and tune and coupling) shifts. If during these states the tunes and couplings are not kept away from harmful resonances, emittance growth and/or beam loss will result.

Closed orbit and quadrupole variations also affect the chromaticities. These effects are usually small and do not pose problems for beam stability. However, persistent current effects in the bending dipoles of superconducting accelerators have a large effect on the sextupole moment. During the 1-3 hour TEVATRON injection front porch, the chromaticities change by as much as 70 units. These changes are undone in the first seconds of acceleration. If the chromaticities are too low during these states, head-tail instabilities may develop. If they are too large, the chromatic tune spread may overlap harmful resonances. In both cases, emittance growth and/or beam loss may result.

In the TEVATRON, control of the tunes, couplings, and chromaticity has been accomplished with open loop control. The currents in the relevant magnetic circuits are specified at sets of discrete breakpoints during each state. Linear interpolation is used between breakpoints. There are several shortcomings to this approach. To have sufficient time resolution, the breakpoints must be spaced closely in time. Since the values of the circuits at the breakpoints must be measured experinentally, determination of the tables of values can be a laborious process. The tables are also not robust against changes in accelerator conditions, such as small closed orbit changes. In addition, the values for the tune and coupling circuits are all dependent upon the closed orbit, and if it changes, all entries must be changed. The breakpoints are 5-10 seconds apart, and one observes tune variations of \pm 0.005 between breakpoints. These variations consuine an appreciable fraction of the TEVATRON working space of 0.025 .

A realtime feedback system could eliminate these problems. Reliable realtime measurements of beam parameters would be used as the inputs to a microprocessor. The micropro-

\footnotetext{
${ }^{1}$ G. Dugan, Particle Accelerators 26, 12 (1990)
} 
cessor contains a table of desired values for these parameters and a model of the accelerator that allows the calculation of the current changes in the circuits to correct an error signal (the difference between the table value and the measured value). This system would overcome the two limitations of a breakpoint system. The feedback bandwidth can be large enough so that corrections can be applied at $60 \mathrm{~Hz}$ or greater, and slow variations in the accelerator conditions can be automatically compensated. A feedback system would be used in conjunction with the tables noted in the previous paragraph. The table values would be set initially, and feedback would make only small changes to eliminate variations between breakpoints and to compensate for changes in accelerator conditions.

The $\mathrm{CBA}^{2}$ system at the Fermilab TEVATRON was designed and built for these uses. It operates as a finite state machine in which each state corresponds to a specific TEVATRON process. Feedback within a state is performed by periodic calls to the state's data acquisition and feedback routines. Each state also includes a set of buffers in which the measurements and calculations from the feedback routine are stored. All data are available through the Fermilab accelerator control system. We will describe the general features of the microprocessor software, the way in which it has been incorporated into the TEVATRON control system, and some of the ways in which it has been used.

\section{General Features of the Feedback System}

The tirne-sequenced operations in a hadron collider dictated our choice of a state machine in which each state corresponds to a distinct accelerator operation. The complete sequence of operations for a state is as follows:

1. Receipt of Timing Signal in the Transition Table - This triggers a call to the the entry routine of the new state. A buffer is allocated from the new state's pool and a time-ofday stamp is filled with the current system date and time. A counter to measure time while in the state is also started.

2. Periodic Calls - The state's periodic routine is called in accordance with a set of periodic rules. The routine calls the data acquistion and feedback routine for the particular state.

3. Exit - Upon receipt of a signal indicating exit from the state, the exit routine is called. This closes the buffer and zeroes the output to the power supplies.

The hardware standard is the Intel Multibus $\mathrm{II}^{3}$ chassis using the Intel 80386 microprocessor. The processor consists of one Micro Industries 80386 processor card with 8 MBytes

\footnotetext{
${ }^{2}$ CBA is an acronym for Colliding Beam Adrastus. See R. Graves, THE GREEK MYTHS, vol. 2, Viking Penguin Inc., New York, New York, 1955, p. 377.

${ }^{3}$ Multibus and iSBX are trademarks of the Intel Corporation
} 


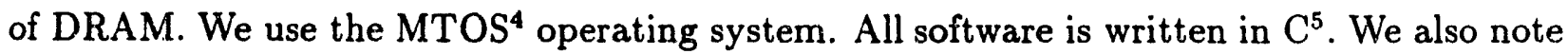
that the software system we will describe was originally written and debugged on a VAX computer, and also runs on VME systems.

The heart of the finite state machine is a set of states with a transition table and a set of periodic rules and buffers for each state. These structures are illustrated in Figs 2 and 3. The transition table consists of a list of allowed transitions between states and the trigger which will cause a given transition. Each state can have transitions into up to 8 other states, although a given trigger signal can occur only once in the transition table for a state. The periodic rules consist of a specification of 8 frequencies and timing signals which will cause the periodic routine to be called. The frequencies vary from state to state, depending upon the accelerator processes occurring. For instance, during acceleration the persistent current effects are removed rapidly, and a frequency of several $\mathrm{Hz}$ is indicated, but during the squeeze the quadrupole circuits change very slowly and a frequency of $1 \mathrm{~Hz}$ is sufficient.

Associated with each state is a set of buffers. The buffer structures consist of a specification of the number of fields, number of records, number of instances of a state which may be buffered before they are overwritten, time-of-day stamps for each buffer, and the data buffers themselves. One buffer is filled for every instance of a state, and after the entire set has been filled, they are overwritten in a circular manner. The number of buffers for each state has been chosen to be large enough so that there is no danger of buffers being overwritten before they have been archived, if desired. The time-of-day stamp is the clock date and time at which the instance of the state occurred. It is stored in a separate circular buffer which maintains the one-to-one correspondence with the data buffers. Each data buffer consists of fields into which the input data, accelerator parameters, and output data may be loaded. A new record consisting of data for all the fields is added to the buffer each time the periodic routine is called.

The computations for each state are performed in the entry, exit, and periodic routines. The entry routine allocates the next available buffer and zeroes the timer which counts time since the start of the state. The exit routine closes the buffer and zeroes the feedback output. The periodic routine consists of routines that read the beam and accelerator parameters, such as the betatron tunes and bending bus current. It also accesses the tables of desired values for beam quantities as a function of time since entry into the state. The desired values and measured values are inputs to the feedback routine, which calculates an error signal. The accelerator model is then used to calculate the feedback current required to null the error signal. These corrections are output in real time to the power supplies and are stored, along with the input data and whatever calculations are desired, in the buffer being used for the particular instance of the state.

We have also provided a facility to perform digital filtering on the input measurements. A task or set of tasks runs continuously and reads the beam parameters. Currently we have implemented only an instantaneous value (no filtering) and simple averaging over a specified interval with input values more than $25 \%$ away from the current average value discarded. Both the frequency of averaging and tolerance are parameters and are chosen to match

\footnotetext{
${ }^{4}$ MTOS is a trademark of Industrial Programming, Inc.

${ }^{5}$ D. Herrup et. al., Real-Tirne Feedback Control of the Tevatron Tunes and Chromaticities, Proceedings of the $2^{\text {nd }}$ European Particle Accelerator Conference, Nice, June 12-16, 1990
} 
the bandwidth of the input signal. These filtered measurements are used by the feedback routines. The accelerator parameters are noise-free. A single reading is sufficient.

To reduce the effects of input noise on the feedback loop, we use the following algorithm to calculate corrections: a tolerance is applied to the error signal. For error signals within 1 tolerance, no correction is applied, and for larger error signals a quantized error signal equal to ( ( \# of tolerance \pm 0.5$)^{*}$ tolerance, depending upon the sign of the error signal) is used as the input to the feedback calculation.

\section{The TEVATRON Implementation}

The features discussed in the previous section are desirable in an operational feedback system for a hadron collider. In our implementaion we incorporated them within the existing features of the TEVATRON control system whenever possible ${ }^{6}$

Fig. 4 is a logical diagram of the complete system using feedback control and the open loop breakpoint tables, and Fig. 5 is a schematic diagram of the system from input measurements to realtime outputs. There are three input paths.

Currently, the realtime accelerator measurements are made using a set of analog signals from the TEVATRON tune trackers. These are a set of phase lock loops which lock to a betatron oscillation in the outputs of a Schottky detector system. Without coupling between the horizontal and vertical betatron oscillations, the tune trackers have an accuracy of \pm 0.002 with a $15 \mathrm{~Hz}$ bandwidth ${ }^{7}$, depending upcn the TEVATRON operating point. They also show a modulation of up to 0.002 caused by the stray magnetic fields of the Main Ring which shares a tunnel with the TEVATRON. The tune trackers also provide a status bit which indicates whether the loop successfully "locked" on a signal. We convert these signals using a 16 channel, 12 bit adc connected to the 80386 processor board through the iSBX connector. The tune tracker signals are averaged as described in the previous section, and both the instantaneous and average values are available through Fermilab ACNET console applications. We also monitor the $A D C$ by digitizing the \pm 5 volt levels in the crate. If they do not have the proper values, a warning can be transmitted to the accelerator operators. The digitized data are filtered as described in the previous section. The input configuration we have chosen was dictated by the signals available, but we could have just as easily used a set of Multibus II input registers for digitized data, or a Multibus II digital signal processor whose output would be available to the 80386 over the bus.

The 80386 accesses data on three TEVATRON-wide data links. TCLK (Tevatron CLocK) ${ }^{8}$

\footnotetext{
${ }^{6}$ D. Bogert, "The Fermilab Accelerator Control System", Proceedings of the Second International Workshop on Accelerator Control Systems, Nuclear Instruments and Methods, A247 (1986),p 133

${ }^{7}$ D. Martin et. al., "A Resonant Beam Detector for TEVATRON Tune Monitoring,", Proceedings of the 1989 IEEE Particle Accelerator Conference, March 20-23, Chicago, IL and J. Fitzgerald and R. Gonzalez, Tune Trackers for the Fermilab TEVATRON, Proceedings of the 1991 IEEE Particle Accelerator Conference, San Francisco, May 6-9, 1991

${ }^{8}$ D.G. Beechy and R.J. Ducar, "Time and Data Distribution Systems at the Fermilab Accelerator", Nuclear Instruments and Methods in Physics Research A247 (1986), p. 231
} 
and MDAT (Machine DATa) are the links which broadcast the timing information and accelerator parameters that the system requires. Initiation of states is done by broadcasting specified clock events on the TCLK link. These clock events are used in the transition tables. The TCLK decoder was specially designed on an 80186-based Multibus II card. It provides signals to any requesting module in the crate based on the occurrence of a TCLK event (or TCLK event + programmed delay) or at a periodic rate of up to $60 \mathrm{~Hz}$. MDAT is received and transmitted on another card. MDAT transmissions consists of identifiers for the various parameters broadcast on the link, followed by the instantaneous value. It is updated at 720 $\mathrm{Hz}$. For our concerns, the most important parameter is the TEVATRON bend bus current. The currents in all of the correction dipoles, quadrupoles, and sextupoles are scaled by this current. We also use the MDAT link to broadcast the feedback corrections to the power supplies to be controlled.

Finally, various types of control data must also be input to the system. These are not realtime signals, and they are downloaded over the Fermilab ACNET control system. The parameters controlling the feedback loops for each state (bandwidths, gains, tolerances, and on/off switches) are downloaded from a dedicated Fermilab ACNET console application. The same application also loads the tables of desired values for the beam parameters.

For tune control, the feedback routine applies the $2 \times 2$ matrix relating changes in tune (the error signal) to the currents in the focussing and defocussing quadrupole busses. The elements of the matrix are determined from a model of the TEVATRON which predicts the current changes needed to produce a given tune change. The instantaneous values of the feedback currents are also accessible from the consoles. The maximum feedback bandwidth of this system is $60 \mathrm{~Hz}$, but for most uses a frequency of $1-15 \mathrm{~Hz}$ is sufficient. The power supplies used in the feedback have bandwidths of greater than $100 \mathrm{~Hz}$, so we expect that the currents will come to their new values before another feedback call is made.

The 80386 processor has two possible output paths. Realtime corrections to power supplies (an identifier and the current change to the supply) are inserted onto the MDAT link. The power st:pply cutput is normally just the value (or interpolated value) from the breakpoint tables described in the Introduction. A power supply controller accesses MDAT, and on decoding its identifier, it adds the MDAT current change to the output taken from its internal tables.

In the other output path, the data buffers are made : ailable to the ACNET console system. We have developed an application to list the contents of the buffers. The user selects the state and incarnation of that state of interest from a menu. He/she then selects the fields to be listed. Currently, there is no graphics capability. It will be added if needed. There is a facility to write buffers to a hard disk and retrieve them for later analysis.

We have conducted several complete closed loop tests of the CBA system. During the setup for a TEVATRON $p \bar{p}$ store, we programmed a table of desired values for the horizontal tune, and activated the system. The horizontal tune was ramped from 20.572 to 20.568 and back. The vertical tune was held constant at 20.581. In Fig. $6 \mathrm{a}$ we plot the horizontal tune tracker signal and the ramp being used, and in Fig. $6 \mathrm{~b}$ we plot the feedback corrections in the focussing a id defocussing quadrupole strings. The ramp took 25 seconds to complete, and each leg was 4-5 seconds long, so the problem wis really a static problem and a gain of 1 and feedback bandwidth of $0.5 \mathrm{~Hz}$ were adequate. As can be seen, the horizontal tune 
tracks the programmed value to within \pm 0.002 , and the vertical tune is held constant.

This first test shows that CBA can regulate according to a programmed ramp during which only the tune circuits are changed. We have also done a test which indicates that CBA can compensate effects caused elsewhere in the TEVATRON. We introduced a single horizontal dipole kick of varying magnitudes at a particular location. In Fig. 7a, we plot the horizontal and vertical tune signals, the current in the dipole creating the kick, and the position at a beam position detector. The kick was increased in steps of $0.25 \mu$ radians and caused a horizontal tune shift of \pm 0.005 at its maximum value. Fig. $7 \mathrm{~b}$ is a repetition of this experiment, but with CBA turned on and with a gain of 1 and bandwidth of $0.75 \mathrm{~Hz}$. We used a tolerance of 0.001 . CBA was clearly able to regulate the horizontal tune to within the tolerance.

Uses

There are many operational uses for this system. It can be used to provide closed loop feedback whenever there is a set of reliable input signals and a well-understood model for the accelerator. The most obvious uses are for tune, coupling, and chromaticity control. In superconducting accelerators, it can be used in conjunction with a magnetic probe meisuring the sextupole moment in the dipoles to correct the persistent current effects. It can also be used for open-loop compensation of any effects which are reproducible, such as the rapid changes in the persistent current sextupole moment at the start of acceleration.

CBA provides an easy way in which to improve the values in the breakpoint tables for the various magnetic circuits. The breakpoints are all indicated by TCLK events, and they can be used to force an entry to a state's periodic routine. These entries can be flagged in the buffers, and one can modify the breakpoint tables to reflect slow drifts in accelerator conditions. Beam aborts also appear on TCLK, and a a snapshot of beam conditions immediately after an abort can be made.

A program has been developed in the TEVATRON for making lattice function measurements by introducing closed orbit bumps and measuring the position changes at the beam position monitors. This technique relies upon the betatron tune not changing as the bump is turned on. However, the higher order fields in the dipoles can create large tune shifts as the orbit changes. CBA will easily handle these relatively slow changes. The orbit bump described in the previous section was taken as part of this program. 


\section{Conclusions}

We have built a general purpose microprocessor based feedback system which has been tailored to fit the finite state behavior of hadron collider operation. The system will work with any reliable realtime inputs and will provide feedback at up to $60 \mathrm{~Hz}$. Each state has it's own feedback routines which contains a model of that particular accelerator state. We have provided a buffer system which matches the states of the state machine and which contain all informaiion describing the operation of the feelback loop. Closed loop corrections to the power supplies can be output on any accelerator-wide link. This system has been tested and shown to work in circumstances which are useful to accelerator operations.

\section{Acknowledgements}

We would like to thank the many people who have contributed to this project. Among them are R. Ducar, J. Zagel, P. Lucas, J. Smolucha, M. Glass, P. Smith, R. Koldenhoven, R. Marquardt, R. Crouch, and B. Hendricks of the Accelerator Division Controls Group, J. Fitzgerald of the Instrumentation Group, and Bob Jushel, G. Annala, and D. Finley of the Main Accelerator Department. Glenn Goderre provided important assistance during the beam tests. D.H. wishes to thank Professor Nicole Jordan of the University of Illinois at Chicago for many useful discussions. This work was supported by the Universities Research Association Inc., under Contract DE-AC02-76CHO3000 from the Department of Energy. 


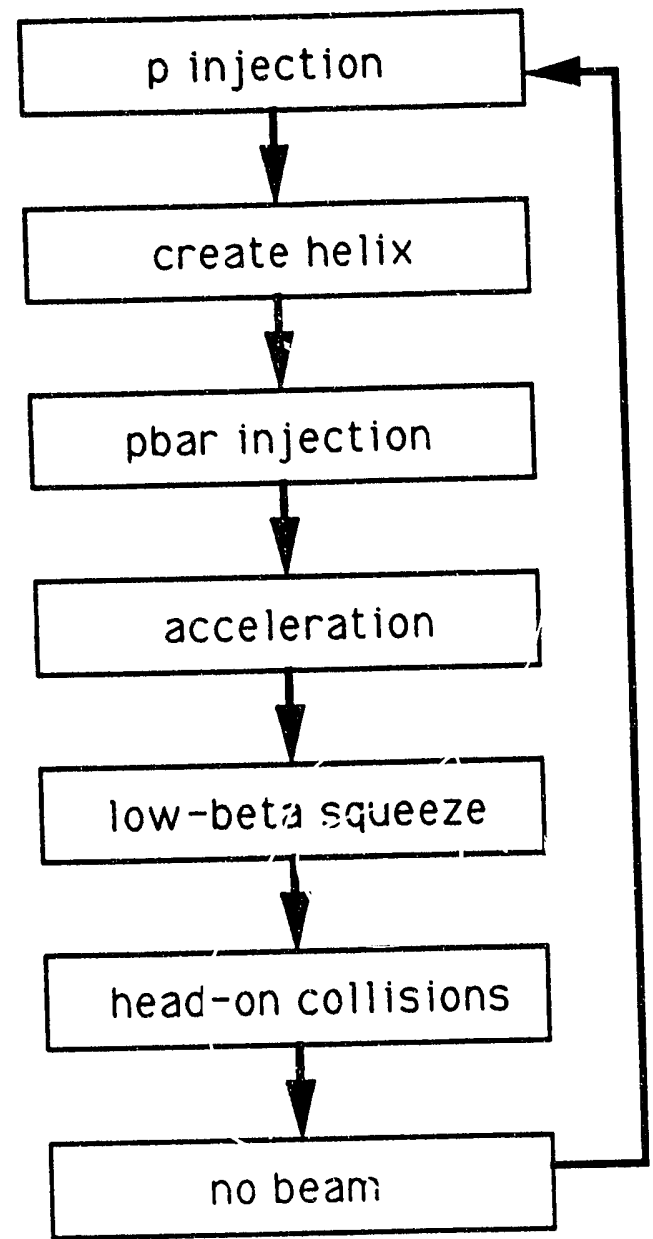

Figure 1. The sequence of operations involved in TEVATRON collider running. 


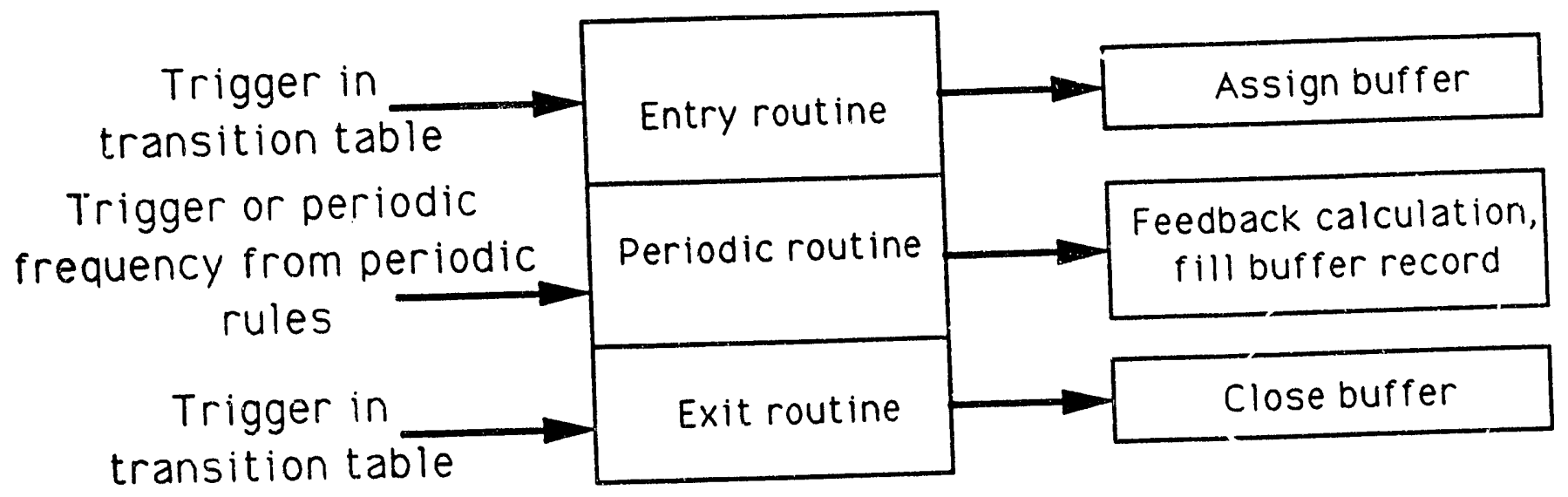

Figure 2. State structures for a CBA state. 


\begin{tabular}{|c|}
\hline Number of instances \\
\hline Number of records \\
\hline Number of fields \\
\hline Circular buffer pointer \\
\hline Time of day $* 1$ \\
\hline Time of day $* 2$ \\
\hline Time of day $* 3$ \\
\hline Time of day $* 4$ \\
\hline Time of day $* 5$ \\
\hline Data buffer $* 1$ \\
\hline Data buffer $* 3$ \\
\hline Data buffer $* 4$ \\
\hline Data buffer $* 5$ \\
\hline
\end{tabular}

Figure 3. Buffer structures for one state with a circular buffer 5 deep. 


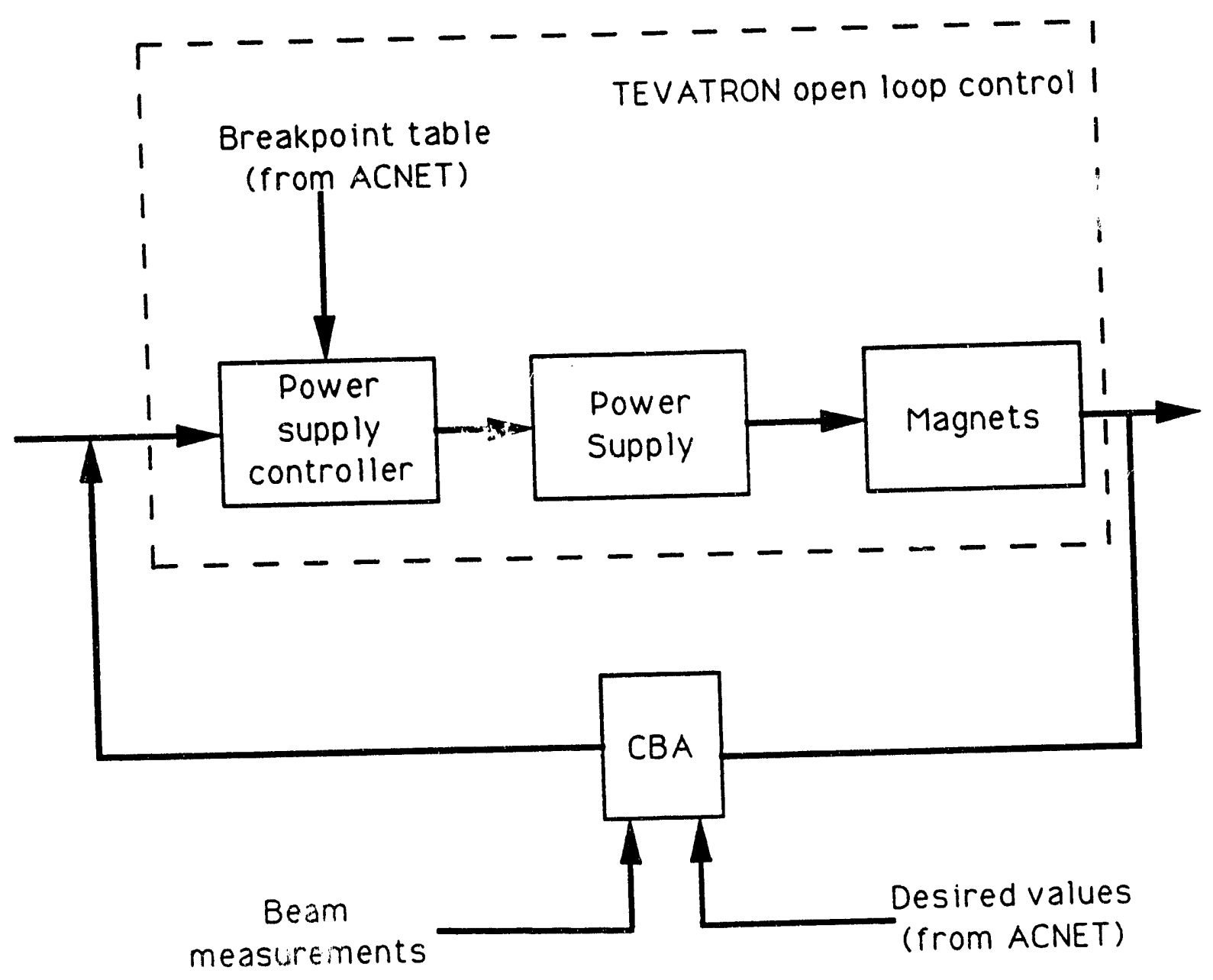

Figure 4. Schematic of the complete CBA closed loop system. 


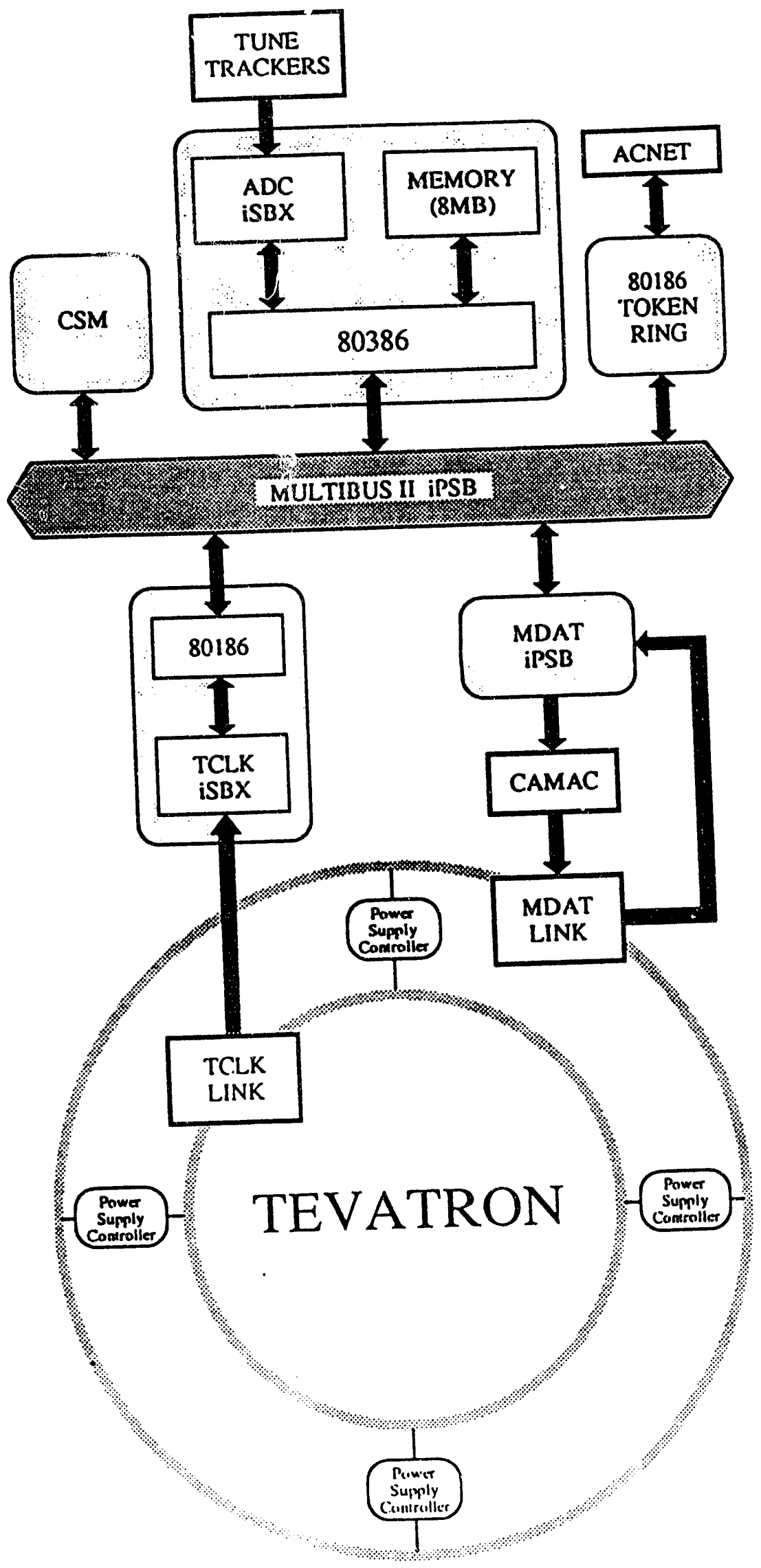

Figure 5. Complete CBA implementation. 


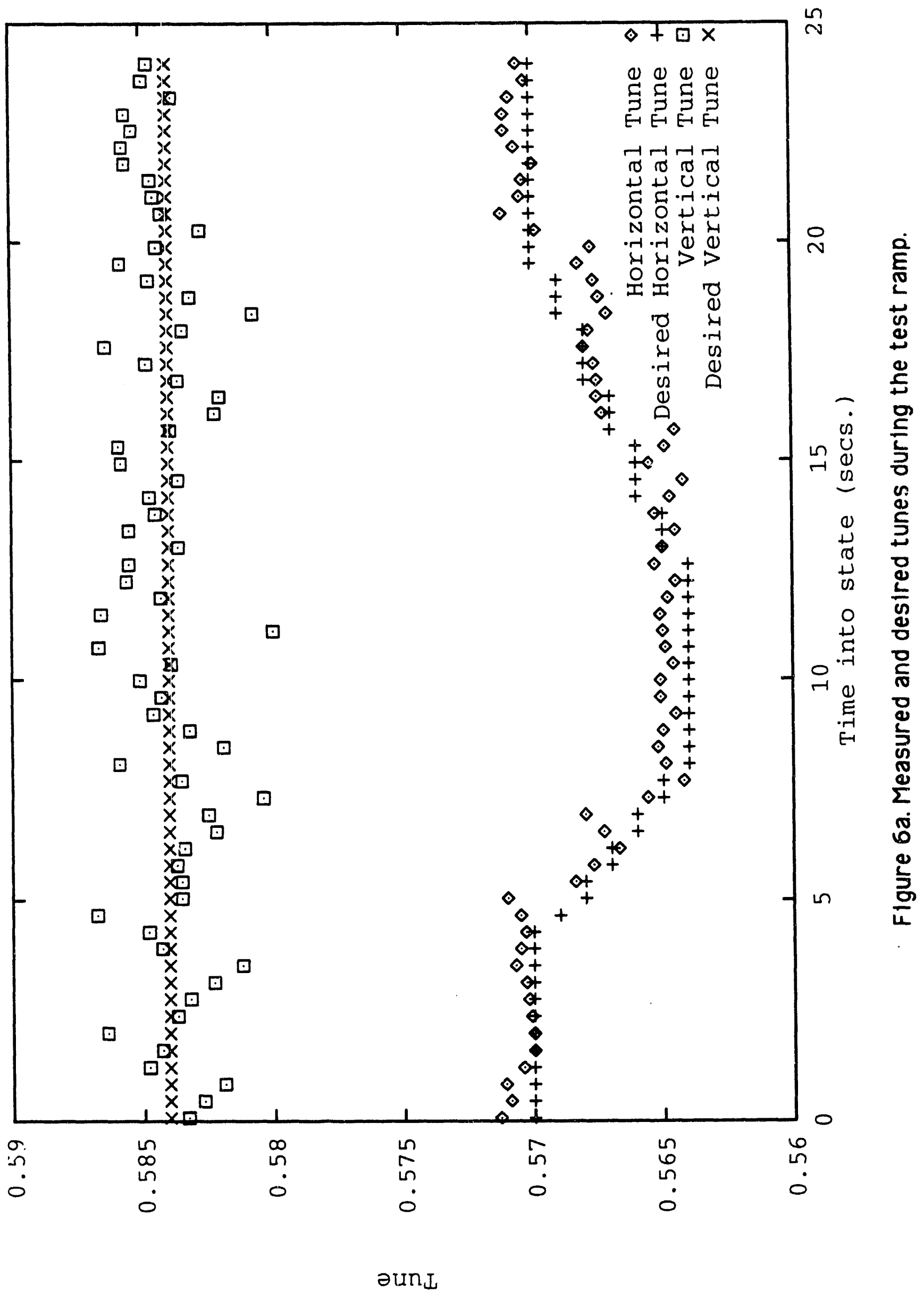




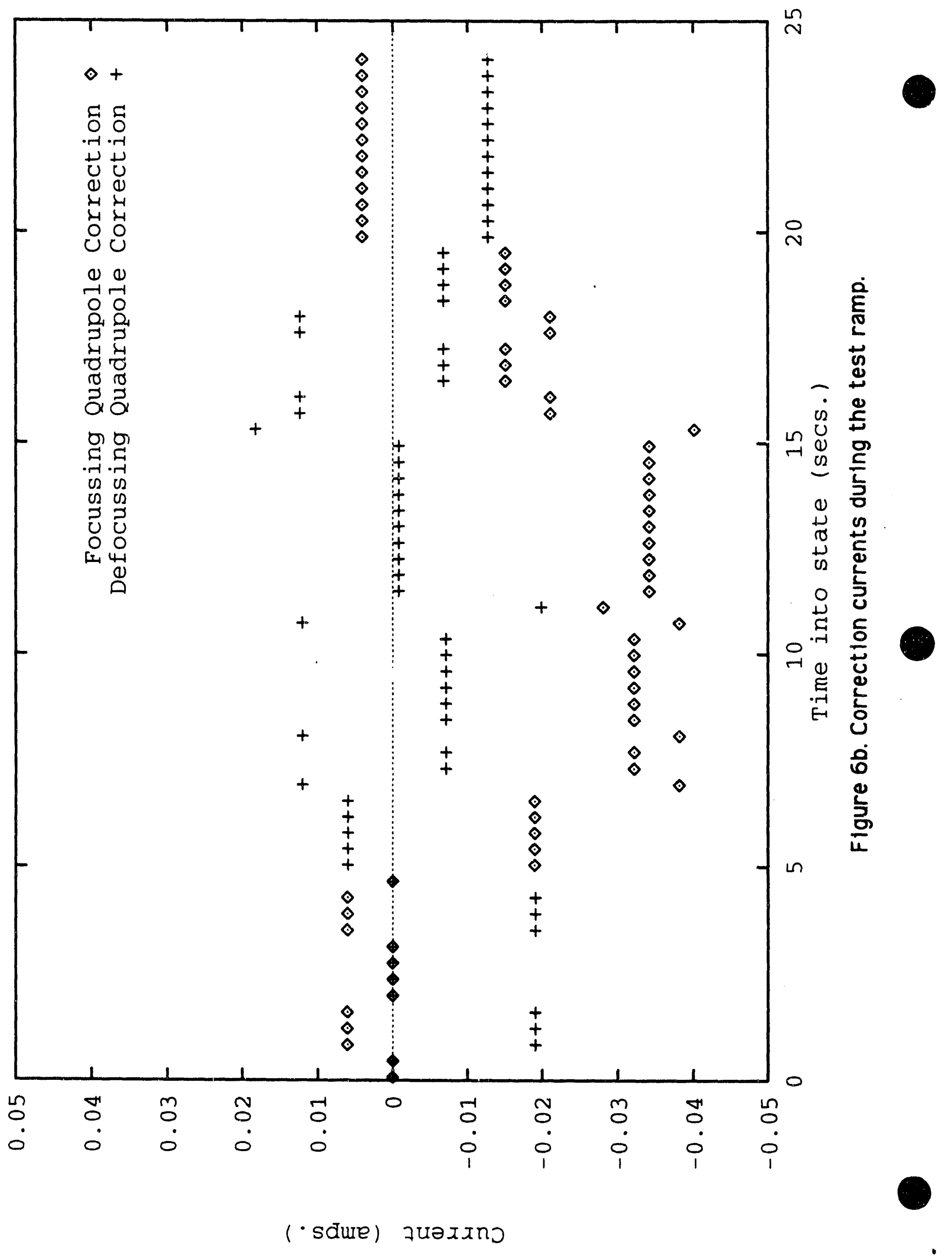




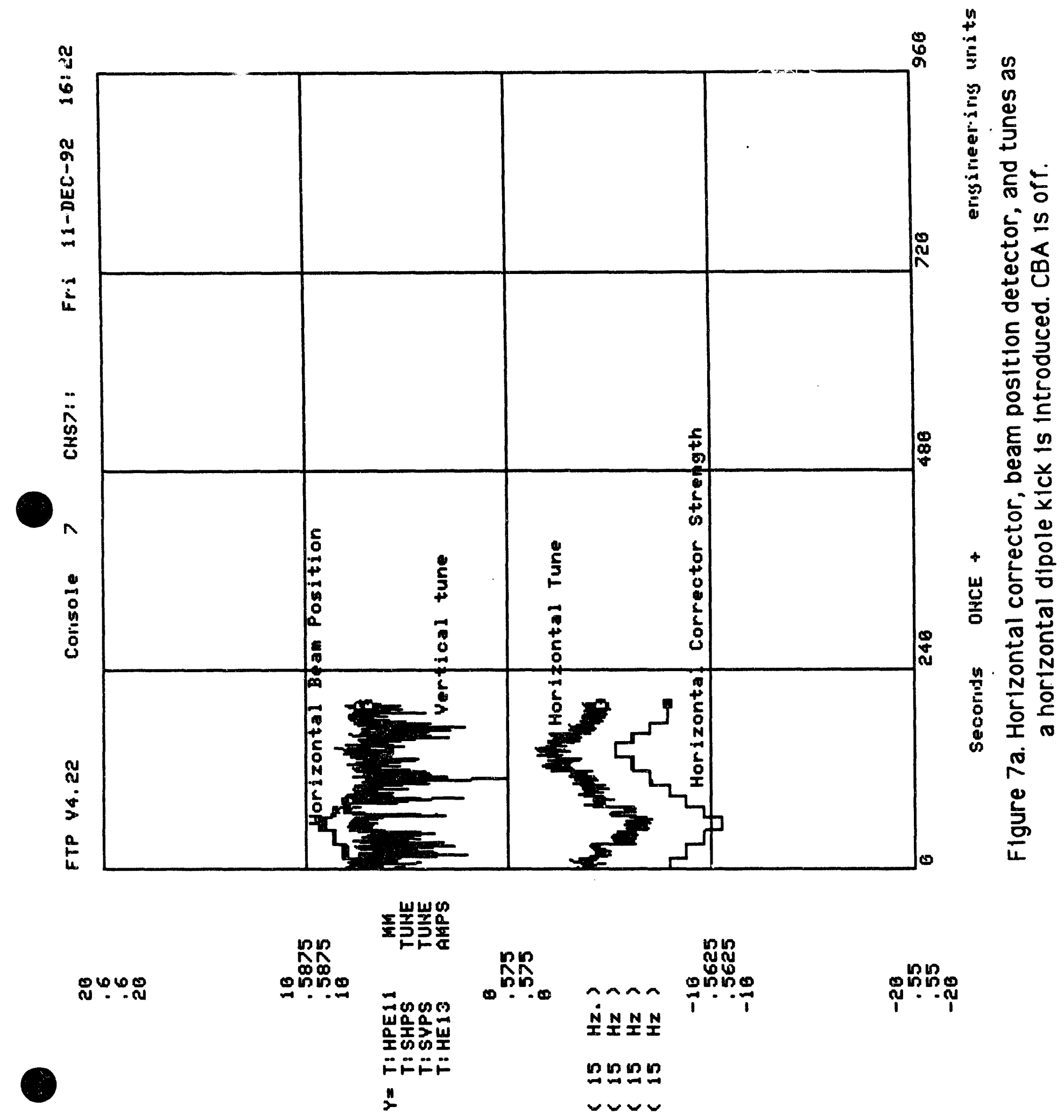




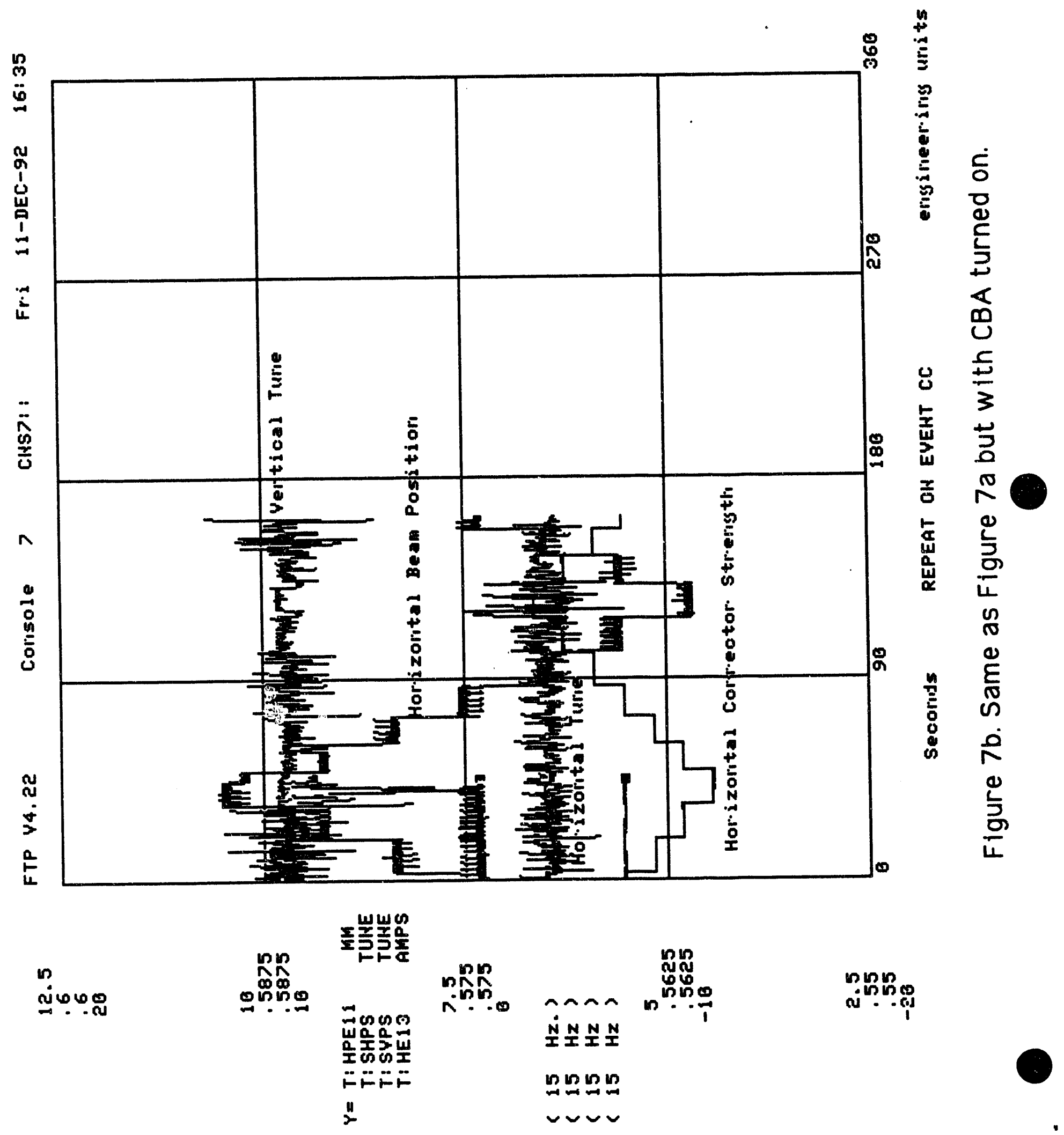



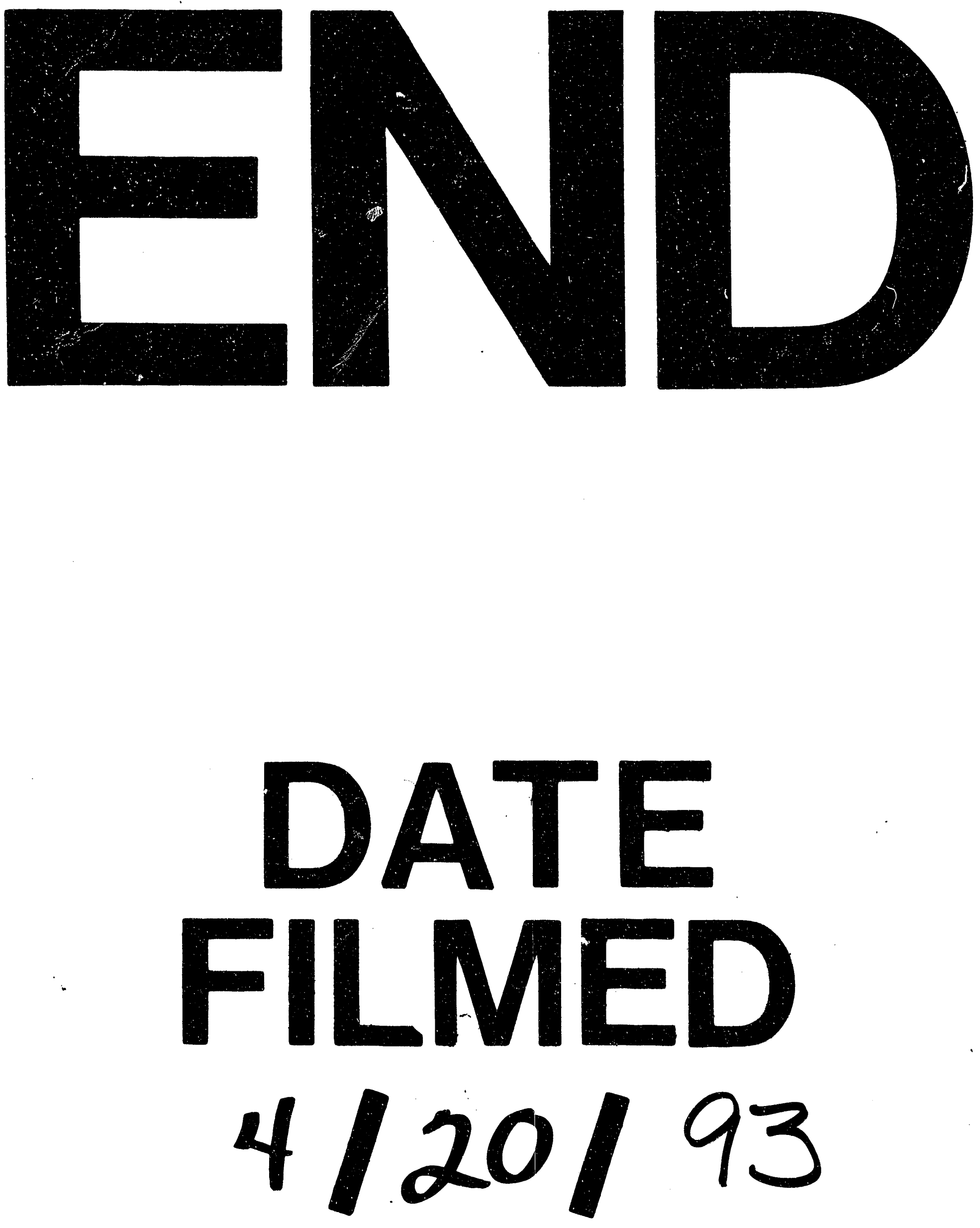

I 


\section{. . . " . " " "}

